\title{
molecules
}

ISSN 1420-3049

http://www.mdpi.org

Review

\section{Phase I and Phase II Ocular Metabolic Activities and the Role of Metabolism in Ophthalmic Prodrug and Codrug Design and Delivery}

\author{
Abeer M. Al-Ghananeem ${ }^{\dagger}$ and Peter A. Crooks* \\ Department of Pharmaceutical Sciences, College of Pharmacy, University of Kentucky, Lexington, \\ Kentucky 40536, USA; ${ }^{\dagger}$ e-mail: amalg0@email.uky.edu
}

* Author to whom correspondence should be addressed; E-mail: pcrooks@email.uky.edu

Received: 10 February 2007; in revised form: 5 March 2007 / Accepted: 5 March 2007 /

Published: 8 March 2007

\begin{abstract}
While the mammalian eye is seldom considered an organ of drug metabolism, the capacity for biotransformation is present. Compared to the liver, the metabolic capabilities of the eye are minuscule; however, phase I and phase II metabolic activities have been detected in various ocular structures. The careful consideration of ocular tissue metabolic processes within the eye has important implications for controlling the detoxification of therapeutic agents and for providing the potential for site-specific bio-activation of certain drug molecules, thus enabling significant improvements in drug efficacy and the minimization of side-effect from either local or systemic drug delivery to the eye. Knowledge of these processes is important to prodrug and codrug development and to researchers involved in the design, delivery and metabolism of ophthalmic drugs. This present article reviews the progress in ocular prodrug and codrug design and delivery in light of ocular metabolic activities.
\end{abstract}

Keywords: Ocular metabolism, drug delivery, drug design, phase I biotransformation, phase II biotransformation.

\section{Introduction}

One might consider the eye an ideal, easily accessible target organ for drug delivery. However, the eye is, in fact, well protected against absorption of foreign materials (i.e. drugs and xenobiotics), first 
by the eyelids and tear-flow, and then by the cornea and conjunctival epithelial barriers of the eye [1]. Furthermore, although the mammalian eye is seldom considered an organ of drug metabolism, in this respect, phase I and phase II biotransformation activities have been detected in various ocular structures. These metabolizing capabilities of the mammalian eye are unique in providing protection from xenobiotics and foreign compounds.

A drug applied to the surface of the eye may cross ocular-blood barriers where it may encounter metabolizing enzymes and cellular transporters before it distributes to the site of action. Other problems of drug delivery to the eye include: poor bioavailability of ophthalmic solutions, clearance and drainage from the eye (ophthalmic drugs typically achieve $<10 \%$ ocular bioavailability), side effects resulting from ophthalmic drugs reaching the systemic circulation, decreased visual acuity, and patient compliance.

Currently there is considerable interest in ophthalmic drug design based on strategies to improve ophthalmic drug delivery through metabolic activation. As ocular enzymes and transport systems are better characterized, their properties become an integral consideration in drug design and development. Various review articles have been published to date describing the strategies employed for enhancing ocular drug delivery [2-6] but only a few studies have addressed phase I and phase II ocular metabolic activity and their utility in metabolism-focused, ophthalmic-specific drug design. This specific area is the focus of this review.

\section{Anatomy of the Eye}

\section{1a. Blood Flow and Membrane Properties of the Eye}

Most ocular tissues have a rich blood supply, except for the cornea and lens. The retina receives its blood supply from the retinal artery, which enters the eyeball along the optic nerve. The outer layer of the retina is adherent to the choroids, which is a highly vascular tissue that supplies the outer segment of the retina, as well as the rest of the optic structures with nutrients and oxygen; this is known as the uveal blood supply [7].

Because of this rich blood supply, the eye must have a way to regulate the entrance of circulating compounds and a way to transport metabolic by-products and/or toxicants out of the eye [8]. The blood-ocular barrier, which functions in a similar manner as the blood-brain barrier, restricts the entry of many compounds and thereby maintains homeostasis in the eye. The blood-ocular barrier can be separated into two distinct barriers; the blood-vitreous barrier and the blood-retinal barrier. The bloodvitreous barrier includes the ciliary epithelium, retinal pigment epithelium, and blood vessels of the retina. The blood-retina barrier involves primarily the retina.

\section{1b. Transport and Distribution Mechanisms in the Eye}

Blood-ocular barriers are important for reasons other than just the removal of toxic waste products and xenobiotics. The eye requires nutrients just like any other organ in the body, and thus requires selective transport mechanisms. There are three major sites of transport for polar nutrients into the eye: the uvea, which consists of the ciliary body and iris, the retina, and the lens [8]. Several studies have 
been conducted to determine the degree of specificity of ocular transport systems. Common transport systems are known to exist for structurally similar amino acids; for instance L-phenylalanine and Lleucine share a common transport system. This may be a mechanism by which many xenobiotics are transported into the eye, as well as a way to deliver pharmacological agents to the eye for the treatment of ophthalmic disorders.

The eye is usually overlooked in tissue distribution studies during drug development. Although the levels of drug and/or metabolite are usually low in most ocular tissues, due to the presence of the blood-ocular barrier discussed above, there are several examples of peripherally administered drugs causing side effects in ocular tissues. Chloroquine is an example of an antimalarial drug that affects the cornea after oral administration, resulting in intolerance to glare and halos around lights [9]. Another drug, chlorpromazine, results in lens deposits and pigmentary retinopathy after systemic administration, [10]. Tamoxifen administration also results in corneal deposits; however, this is due to its insolubility in tear solution. The active metabolite of acetaminophen, $N$-acetyl-p-benzoquinone imine, has been found to bind to ocular tissues, resulting in cataract formation [9].

The mode of administration of a drug can be an important factor in determining ocular tissue distribution. Studies of single dose parenteral administration of pentobarbital, aspirin, and three antibiotics showed that the highest drug levels were found in the cornea and iris, and in some studies in the retina and conjunctiva. Drug concentrations in the choroid, sclera, and retina were intermediate, with the aqueous and vitreous humors and lens tissue having the lowest concentrations. There is a serious lack of data on drug/metabolite concentrations in the ciliary body after parenteral drug administration, an ocular tissue that is known to concentrate drugs, as well as having marked metabolizing capability. Studies carried out after topical application of drugs have revealed that the ciliary body is usually intermediate, and in one case, is low in drug concentration [8].

In elimination studies, several drugs have been shown to clear more slowly from the eye compared to other tissues [11]. The iris, retina, choroids, and uveal tract are the tissues that result in most drug accumulation, and slow elimination has been associated with drugs binding to melanin, the pigment epithelium.

\section{Metabolic Activity in the Eye}

\section{2a. Phase 1 metabolic activity in the eye}

Because the eye is an important site of exposure to xenobiotics, it is not surprising that it would possess enzymes capable of metabolizing such foreign species. The eye displays a regional distribution of Phase 1 metabolic activities toward endogenous and xenobiotic substrates. In general, these activities are highest in those ocular structures adjacent to regions of highest uveal blood flow. Cytochrome P-450 activity has been identified by characteristic differential carbon monoxide-binding spectra; the difference in spectra obtained between carbon monoxide bound and reduced microsomes of bovine ciliary body and cornea [12,13]. In these studies microsomes were prepared from bovine ciliary bodies. The cytochrome P-450 contents and related components of the microsomal electron transport system have been determined to be 32 pmoles/mg protein, which is about $4 \%$ that found in rat liver. 
Cytochrome P450-dependent metabolism of endogenous substrates (arachidonic acid, prostaglandin and steroids) in the eye has been demonstrated [14,15]. P-450-dependent arachidonic acid was found to primarily occur in the corneal epithelium, but detectable activity was also found in the retina, retinal pigment epithelium and ciliary body [14]. Prostaglandin hydroxylase-(omega/omega1) activities were detected by Asakura and Shichi primarily in ciliary body epithelium [15]. Research by Shichi et al, generated the earliest reports documenting the presence of cytochrome P-450dependent aryl hydrocarbon hydroxylase activity in the eye $[16,17]$. Further research by this laboratory and others demonstrated that ocular tissues display substrate-dependent differences in activities in various ocular regions, which is indicative of the presence of multiple forms of cytochrome P-450 distributed heterogeneously $[13,18,19]$.

Table 1 identifies those cytochrome P-450-dependent activities reported to be present in the eye and their location. Schwartzman et al. measured aryl hydrocarbon hydroxylase (AHH), 7-ethoxycoumarin-O-deethylase (ECOD) and benzphetamine demethylase (BPDM) in bovine ocular tissues [14]. These authors reported that the highest levels of AHH occurred in the ciliary body and were five (retinal pigment epithelium) and ten (retina or corneal epithelium) times higher than in other ophthalmic tissues. ECOD activity was highest in the retinal pigment epithelium, then in decreasing levels of activity; ciliary body, corneal epithelium, retina and corneal endothelium. BPDM activity was only detected in retinal pigment epithelium and ciliary body tissue. Cytochrome P-450-dependent activities in ocular tissues are inducible by the "classical" p450 inducers, phenobarbital (PB) and 3methylcholanthrene (3-MC), and by clofibrate and hypoxia. Based on the induction of specific activities by different inducers, the presence of particular cytochrome P-450 isoforms may be deduced [20].

Table 1. Cytochrome P-450-dependent metabolism in ocular tissues.

\begin{tabular}{|c|c|c|c|}
\hline Enzyme & Substrate & Ocular Tissue & Ref. \\
\hline $\begin{array}{l}\text { 7-Ethoxyresorufin-O- } \\
\text { dealkylase (EROD) }\end{array}$ & 7-Ethoxyresorufin & Porcine ciliary body, and bovine ciliary body & 18,10 \\
\hline $\begin{array}{c}\text { 7-Pentoxyresorufin-O- } \\
\text { dealkylase (PROD) }\end{array}$ & 7-Pentoxyresorufin & Porcine ciliary body, and bovine ciliary body & 18,10 \\
\hline $\begin{array}{l}\text { 7-Ethoxycoumarin } O \text { - } \\
\text { deethylase (ECOD) }\end{array}$ & 7-Ethoxycoumarin & $\begin{array}{l}\text { Bovine corneal epithelium, retinal pigment } \\
\text { epithelium, retina, ciliary body and corneal } \\
\text { epithelium }\end{array}$ & 12,14 \\
\hline $\begin{array}{c}\text { Aryl hydrocarbon } \\
\text { hydroxylase (AHH) }\end{array}$ & Benzo(a)pyrene & $\begin{array}{l}\text { Bovine retina, cornea, iris, choroid, ciliary } \\
\text { body; Bovine retinal pigment epithelium, } \\
\text { corneal endothelium; Mouse eye; Chick } \\
\text { embryo retinal pigmented epithelium }\end{array}$ & $\begin{array}{c}12,14, \\
15\end{array}$ \\
\hline $\begin{array}{l}\text { Benzphetamine demethylase } \\
\text { (BPDM) }\end{array}$ & Benzphetamine & $\begin{array}{l}\text { Bovine retinal pigment epithelium, and ciliary } \\
\text { body }\end{array}$ & 12,14 \\
\hline Prostaglandin hydroxylase & Prostaglandin & Porcine iris, ciliary body & 13 \\
\hline Cyclooxygenase & Arachidonic acid & $\begin{array}{c}\text { Bovine and rabbit corneal epithelium; Bovine } \\
\text { corneal epithelium, ciliary body and corneal } \\
\text { endothelium }\end{array}$ & 12,16 \\
\hline
\end{tabular}


Other phase 1 activities in the eye have been detected by immunohistochemical methods or by metabolic activities toward specific substrates. Like the cytochrome P-450 monooxygenases, these other enzyme systems show various levels of activity and heterogeneous distribution throughout the ocular tissues. Monoamine oxidase has been detected by histochemistry in the optic and ocularmotor nerves, ciliary epithelium, pigmented epithelium and the plexiform layer of rabbit retina [21]. Monoamine oxidase activities toward $\beta$-phenylethylamine have been detected in bovine retina [22].

Diamine oxidase activity has been reported to be present in bovine aqueous humor using pdimethylaminomethyl benzylamine as substrate: this activity was not detected in the lens, cornea vitreous or sclera [23]. Kremzner et al. reported the presence of the physiological substrates of diamine oxidase, i.e. putrescine, spermine and spermidine, in the lens [24], despite the lack of activity reported by Crabbe [23] in lens tissues. The hydrolysis of dipivefrin, an ester prodrug of epinephrine, has been reported to occur in rabbit corneal homogenates [25], yielding the active drug.

Sanchez-Chavez et al. studied the acetylcholinesterase (AChE) and butyrylcholinesterase (BChE) activities in the neural retina and retinal pigment epithelium (RPE) of adult rats and showed that amphiphilic tetramers and monomers of AChE are abundant in neural retina, and enzyme tetramers, dimers, and monomers in RPE. It was suggested that the AChE in the neural retina might be involved in cholinergic actions [26].

$\beta$-Glucuronidase activity has been reported in ocular tissues of man, rabbit, and guinea pig [27]. The activities of $\beta$-glucuronidase and other hydrolases in the anterior segment tissues of human, pig and rat eyes were determined and reported in the cornea, ciliary epithelium and iris [28]. These same authors examined hydrolase activities in the trabecular meshwork of normal and glaucomatous eyes; they found increased levels of nonspecific esterases, $\gamma$-glutamyl transpeptidases and aminopeptidases in trabecular from glaucoma patients when compared to controls [29]. It was also reported that increased hydrolase activities occur in the inflamed eye [30].

Oxidoreductases have been found in ocular tissues of many different human sources species. Zetacrystalline, a lens protein in the Guinea pig, exhibits NADPH-dependent oxidoreductase activity with 2, 6-dichlorophenolindophenol [31]. Aldehyde dehydrogenases have been reported to occur in many different tissues of the mammalian eye, including humans. These enzymes occur in many isoforms with different substrate affinities [32,33]. The aldehyde dehydrogenases of the ocular tissues have been proposed to be involved in lipid peroxidation, ethanol metabolism, carbohydrate metabolism and the visual cycle of the retina [34].

\section{2b. Phase II metabolic activity in the eye}

Several studies have shown that various tissues of the eye are capable of Phase II conjugation activity. One of the most comprehensive studies was carried out by Watkins et al. using rabbit ocular tissues [35]. Five tissues of the eye, including lens, choroids, iris, retina, and cornea, were assayed for $\mathrm{N}$-acetyltransferase, glutathione-S-transferase, UDP-glucuronosyltransferase, and 2-naphthol sulfotransferase activities. The iris/ciliary body had the highest glutathione S-transferase activity, while the cornea exhibited the highest specific activities for $N$-acetyl-, sulfo-, and UDP-glucuronosyltransferases. The lens was the only tissue tested that did not have any significant phase II biotransformation capacity. In addition, ocular activity for these Phase II enzymes has been compared 
to that of liver and other extra hepatic organs. The $N$-acetyltransferase activity in the iris/ciliary body was nearly as high as that in liver, kidney, or intestine, whereas the glutathione S-transferase activity in the cornea and iris/ciliary body was 70 and 89\%, respectively, of the activity in the intestine, and sulfotransferase activity of the cornea was greater than the same activity measured in the kidney. These results support the hypothesis that the eye is susceptible to exposure to endogenous and exogenous substrates, and therefore must possess the capacity for detoxification biotransformation. The cornea is particularly susceptible to exogenous exposure to xenobiotics and/or irritants; therefore, biotransformation activity could serve as a first line of defense. It is known that the cornea can tolerate high concentrations of topically applied compounds. Likewise, the lack of metabolic activity present in the lens is not surprising, given the limited blood supply to this tissue. Even though the majority of the protein content exists in the lens, the primary protein of the lens is $\alpha$-crystalline, which has little or no metabolic capability. Glutathione is present in the lens; without which the presence of significant levels of potentially harmful xenobiotics can occur.

Earlier work by Saneto et al. revealed the presence of all enzymes involved in glutathione $S$ transferase activation, i.e. $\gamma$-glutamyl transpeptidase, cysteinylglycinase, and $N$-acetyl transferase, in bovine ocular tissues, including lens, cornea, retina, and retinal pigmented epithelium [36,37].

Ahmad et al. compared the structural relatedness of bovine glutathione $S$-transferase to the three classes of human glutathione $S$-transferase, i.e. alpha, mu, and pi [38]. Using immunoblotting techniques, expression of these isozymes was examined in the lens, cornea, and retina. It was observed that the various isozymes were expressed in a tissue-specific manner, due to the specific functional needs of these various ocular tissues. The individual isozymes of glutathione $S$-transferase are known to carry out particular functions, e.g. the alpha class is thought to be involved in protection of the tissues from oxidative damage via glutathione peroxidase-2 activity. However, Saneto et al. in an earlier study purified two isozymes of glutathione S-transferase from bovine retina and showed that the retina did not contain selenium-dependent glutathione peroxidase activity [37]. $\mathrm{N}$-acetyl transferase activity has been identified in the retina of male Sprague Dawley rats [39]. These authors used tryptamine to demonstrate the presence of $N$-acetyl transferase in retinal homogenates from rat. The rate of tryptamine acetylation varied over a $24 \mathrm{~h}$ period with maximal activity occurring around noon.

Enzymatic step-wise methylation of membrane phosphatidylethanolamine (PE) to phosphatidyl- $N$ methylethanolamine (PME) and then phosphatidylcholine (PC) has been shown to alter membrane properties and responsiveness of cells for activation of receptors by chemical transmitters. The conversion of PE to PME and PME to PC in the presence of $S$-adenosyl-L-methionine (SAM) are both catalyzed by two phospholipid $N$-methyltransferases, PMT I and PMT II, of which PMT I is the rate limiting enzyme. The retina is a good neuronal model for chemical transmission and it was found that rat retina contains both PMTs and fatty acid carboxymethylase (FACM) [40].

\section{Metabolism-focused Ophthalmic-specific Drug Design and Delivery Systems}

As described above, most ophthalmic drugs were not originally developed for the treatment of eyes; such drugs were originally intended for other therapeutic areas and were adopted for ophthalmic drugs by virtue of accidental observation of efficacy in eye disease. For these reasons, there is considerable interest and a need for improved ophthalmic drug delivery strategies. The current trend in ophthalmic 
drug design is based on the drug metabolism properties of the eye, which include the prodrug and codrug approach, retro-metabolic drug design approaches; chemical delivery (CD) and soft drug (SD) systems. In this review article we will discuss recent development in the area of ocular prodrug and codrug design.

\section{3a. The Prodrug Approach}

The prodrug approach is one of the most promising and viable strategies currently being investigated for ophthalmic drug delivery. One aspect of current research in ophthalmic drug delivery is focusing on the design of ophthalmic prodrugs which explore the drug metabolism capability of ocular tissues. The cornea epithelium and endothelium contain 100-fold greater amount of lipid material per unit weight than the hydrophilic stroma [41]. Lipophilic drugs absorb into epithelial tissue, but their partitioning from the epithelium to the stroma may become a penetration-limiting factor [42]. Consequently, adequate solubility both in epithelium and stroma is required. One way to achieve biphasic solubility is to design lipophilic prodrug that can be converted to more hydrophilic derivative or parent drug in the epithelium.

The prodrug approach has been utilized to enhance the ocular delivery of pilocarpine [43-46]. Pilocarpine is a widely used topical mitotic agent used to control the elevated intraocular pressure associated with glaucoma, but the drug itself presents significant delivery problems. The rapid loss of the drug from the precorneal area via drainage, the low lipophilicity, and the poor permeability across the corneal membrane all contribute to the drug's poor bioavailability, where only $1-3 \%$ or less of the topically administered dose gains access to the internal eye structure [47-49]. Furthermore, the current dosage regimen for pilocarpine is 3-6 times per day, due to the drug short half life.

For the above reason, a number of lipophilic alkyl and arylalkyl esters of pilocarpic acid were synthesized [43-46] and evaluated as prodrugs of pilocarpine. The results obtained suggested that the pilocarpic acid esters may be potentially useful prodrugs with enhanced drug delivery and bioavailability properties, especially when further derivatized to give non-labile, easily formulated pilocarpic acid diesters that convert readily to the parent drug in vivo (Figure 1) [43,44]. In aqueous solution the esters undergo a quantitative and apparent specific base-catalyzed lactonization to pilocarpine [50]. The cyclization most likely involves pre-equilibrium ionization at the hydroxyl group and intramolecular nucleophilic attack of alkoxide ion on the ester carbonyl moiety. It was reported that the optimal lipophilicity for improving corneal permeability (i.e., the rate of ocular pilocarpine delivery), was observed at $\log \mathrm{P}$ values of 3-4, but more extensive corneal uptake by the most lipophilic prodrugs suggested that the largest ocular bioavailability may be obtained with larger values of $\log$ PC [51].

The diester prodrugs were of special interest, since they combine an enhanced bioavailability and greatly prolonged duration of action with high stability in eye drop formulations. Some studies suggested that, although the corneal permeability of pilocarpine is increased by prodrug derivatives, ocular irritation is associated with the in vivo use of these lipophilic prodrugs. The eye irritation of pilocarpine prodrugs increased with increasing lipophilicity, $\mathrm{pH}$ and concentration. Such lipophilic pilocarpine prodrugs exhibit amphiphilic properties, which may contribute to the ocular irritation of these compounds [52]. Furthermore, the ocular delivery of pilocarpine prodrugs may be enhanced 
while the local irritation can be minimized by appropriately combining buffer, viscosity and complexation with polymer formulation approaches [53-55]

Topically applied $\beta$-blockers, which decrease aqueous humor formation by ciliary-body processes, are rapidly eliminated from the precorneal area because of their drainage via the nasolacrimal duct, dilution by tear turnover, and absorption into the systemic circulation [56,57]. Therefore, the usefulness of $\beta$-blocking agents in glaucoma has been limited because of respiratory side effects $[58,59]$. An effective approach to improve the ocular delivery of such drugs and to diminish the adverse effects is the use of lipophilic prodrugs. Sasaki et al. have studied the ocular delivery of $O$ acetyl, $O$-propionyl, $O$-butyryl and $O$-valeryl ester prodrug derivatives of the $\beta$-blocker tilisolol, in order to decrease intraocular pressure after instillation [60]. All the above prodrugs showed rapid enzymatic conversion to tilisolol in ocular tissue homogenates. This rapid conversion was reflected in the enhanced delivery of tilisolol through corneal, conjunctival and scleral membranes. These results demonstrate the potential utility of lipophilic prodrugs of tilisolol as anti-glaucoma agents.

Figure 1. Pilocarpic acid diesters prodrugs and their hydrolysis in the ocular tissue.
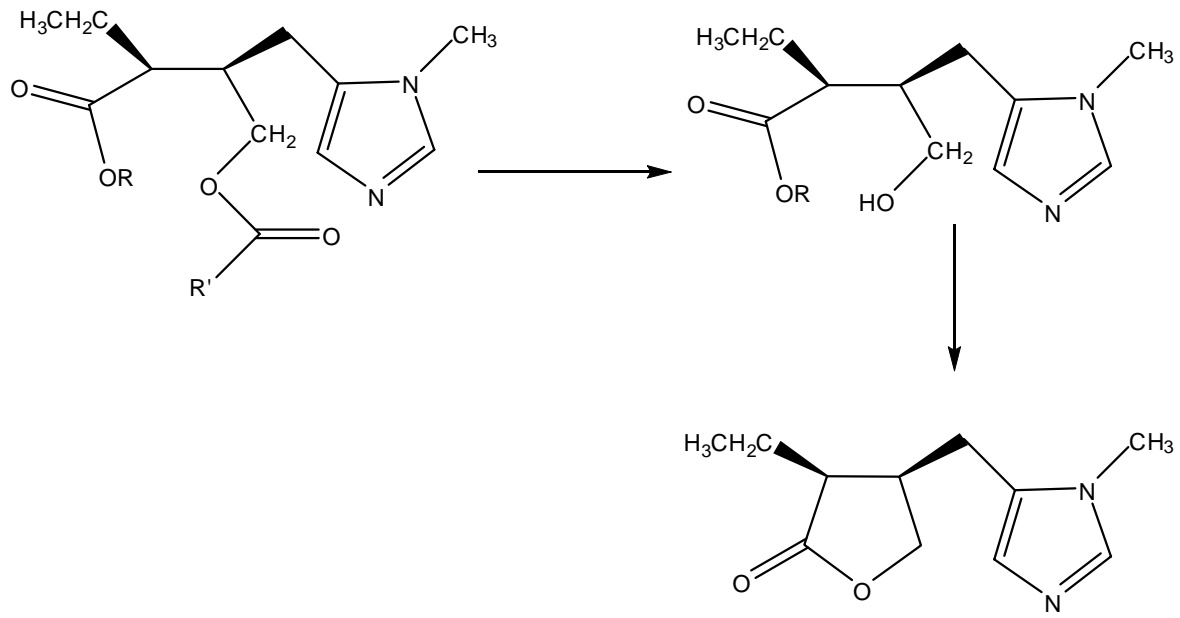

Helbreg et al. investigated the ocular metabolism of bimatoprost (Lumigan ${ }^{\circledR}$ ), the ethylamide prodrug derivative of the potent prostaglandin FP agonist, 17-phenyl-trinor PGF2 $\alpha$, using either human or rabbit ocular tissue [61]. The ethylamide group of bimatoprost was hydrolyzed by rabbit and human cornea, iris/ciliary body and the sclera to produce the parent drug (Figure 2). These studies demonstrated that human and rabbit ocular tissue (cornea, iris/ciliary body and sclera) can convert bimatoprost to the potent prostaglandin FP agonist 17-phenyl-trinor PGF2 $\alpha$.

Figure 2. Bimatoprost prodrug and its hydrolysis to the active form, bimatoprost acid, in the eye.<smiles>CCNC(=O)CCCC/C=C/C[C@H]1[C@H](O)C[C@@H](O)[C@@H]1/C=C/[C@H](O)CCc1ccccc1</smiles>

Bimatoprost

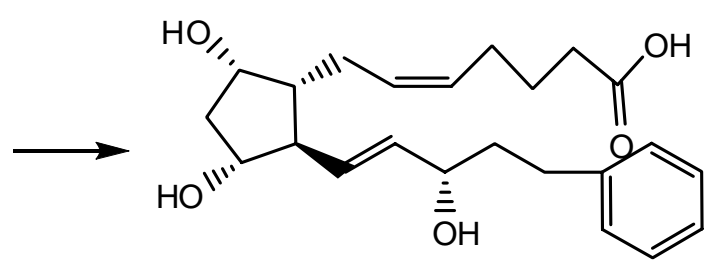

Bimatoprost Acid 17-Phenyl-trinor $\mathrm{PGF}_{2 \alpha}$ 
Enzymatic regeneration of parent drug in ocular tissue from the prodrug derivative is essential to retain activity. Hussain et al. [62] studied the enzymatic regeneration of epinephrine from its prodrug ester in rabbit eye homogenate. As expected, the prodrug itself had no activity, while upon enzymic regeneration of epinephrine from the prodrug the reaction mixture exhibited $\alpha$-adrenergic activity.

Prodrug administration has proven to be an effective method for drug delivery to the anterior segment of the eye. Since the cornea is lipid in nature, it is only logical that it would be more permeable to a lipophilic prodrug compared to a more polar parent drug. The prodrug would then be metabolized by resident enzymes, to the parent compound. Good examples of successfully marketed ophthalmic prodrugs are: dipivephrine for the delivery of epinephrine as the dipivalyl ester of epinephrine, and bimatoprost, latanoprost and travoprost, which are isopropyl ester prodrugs that are prostaglandin $\mathrm{F}_{2} \alpha$ analogs.

\section{3b. The Codrug Approach}

The same concept has also been employed in a novel codrug design to concomitantly deliver two physicochemically different, synergistic drug molecules, which would provide improved physicochemical properties compared to a physical combination of the two parent drugs, and provide controlled drug release upon hydrolysis in tissue $[63,64]$. Codrugs or mutual prodrugs contain a covalent linker tethering two or more synergistic compounds together in order to improve the drug delivery properties of one or both drugs. Like prodrugs, codrugs are bioconvertible derivatives of the component active parent compounds. Covalent linkage of a lipophilic angiostatic steroid such as $3 \alpha$, $17 \alpha$, 21-trihydroxy-5 $\beta$-pregnan-20-one (trihydroxy steroid, THS) to an antiproliferative compound of comparatively higher aqueous solubility, such as 5-fluorouracil (5FU), would afford a hydrolysable codrug with improved physicochemical properties (i.e. intermediate between the water-insoluble THS molecule and the highly water-soluble 5FU molecule) leading to an increase in the bioavailability of both parent drugs with an expected increase in angiostatic efficacy.

A formulation consisting of directly compressed sustained release pellets prepared from material consisting of a molecule of THS covalently linked via carbonate moieties to two molecules of 5FU to form a novel THS-BIS-5FU codrug (Figure 3) for the treatment of angiogenesis and/or retinal detachments resulting from proliferation of fibrovascular networks can be considered as an ocular chemical delivery system for delivery of THS and 5FU upon hydrolysis over extended periods of time. The codrug hydrolysis profile in different media illustrated the successful regeneration of the parent drugs. However, the release of THS was slower than the release of 5FU, since THS generation proceeded through a cyclic carbonate intermediate. Results from these studies provide valuable information for optimization of the codrug design and pelleted formulation of codrugs in order to achieve sustained release for ocular angiogenesis therapy. Hydrolysis kinetics analysis demonstrated no detectable levels of the THS-BIS-5FU codrug which illustrated that the rate limiting step is the dissolution of the codrug from the pellet, then immediate hydrolysis to regenerate 5FU and a cyclic carbonate intermediate that subsequently degrades to THS. [65]. 
Figure 3. The THS-BIS-5FU codrug and the hydrolytic pathway for the release of THS and 5FU.

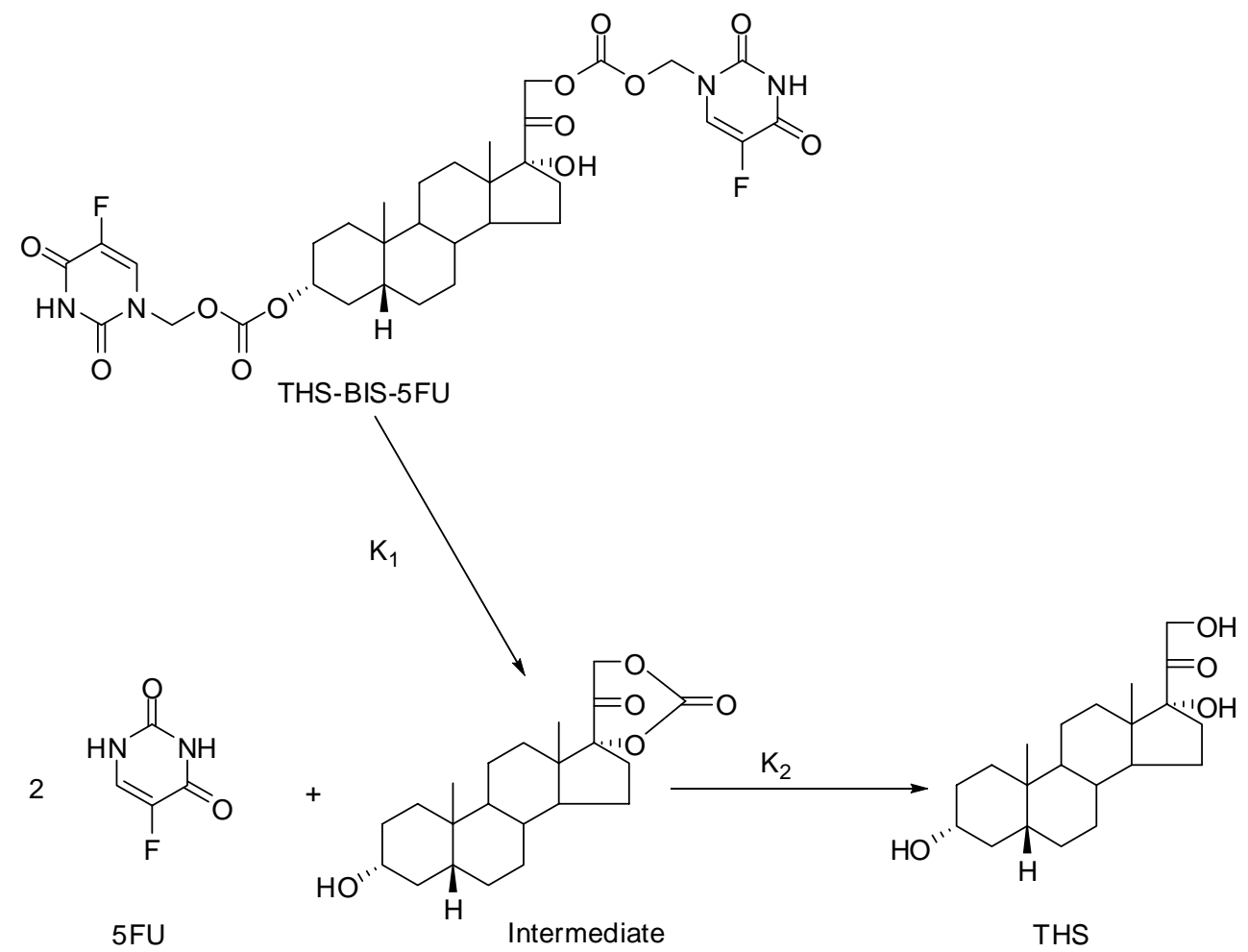

Furthermore, Cardillo et al. have reported on a naproxen-5FU codrug system for the treatment of experimental post-traumatic proliferative vitreoretinopathy. Their results suggested that this codrug system effectively inhibits the progression of experimental proliferative vitreoretinopathy (PVR) in a rabbit trauma model that closely resembles PVR in humans [66]. This unique concept of codrug design has also been utilized in our laboratories for codrugs of ethacrynic acid (ECA) covalently linked to either atenolol (ATL) or timolol (TML) via ester bond linkages (Figure 4) were designed and synthesized to improve ocular delivery, and in addition, to take advantage of the apparent synergistic mechanism of ECA and the two $\beta$-adrenergic receptor antagonists [67]. An important intraocular pressure-reducing agent prostaglandin F2 $\alpha$ (PGF2 $\alpha$ ) has low ocular bioavailability.

Figure 4. The structure of ECA-ATL and ECA-TML codrugs.
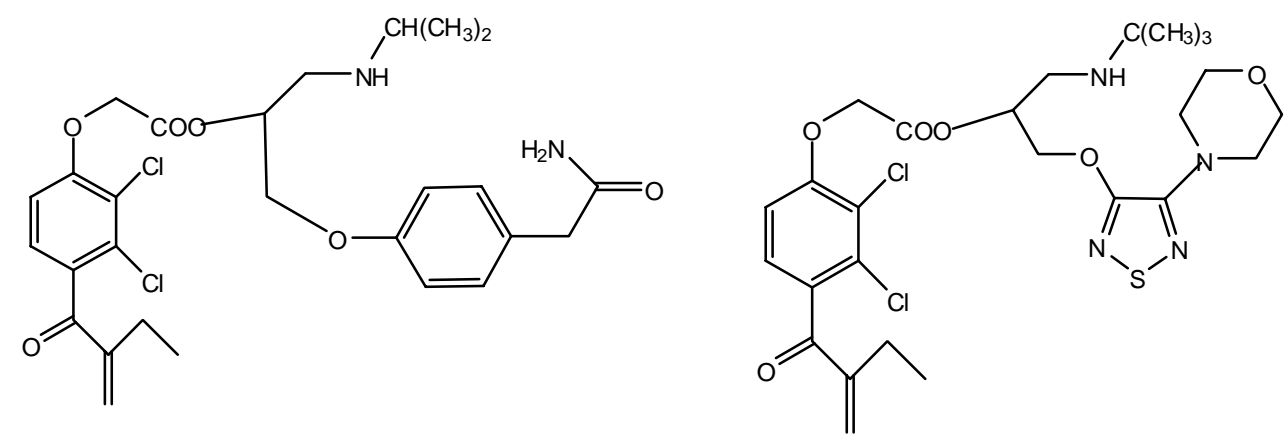

Cynkowska et al. [68] studied a novel codrug containing PGF2 $\alpha$ or PGF2 $\alpha$ triacetate linked to a $\beta$ blocker such as timolol (TML) or atenolol (ATL) via an ester moiety to permit hydrolytic cleavage at physiological $\mathrm{pH}$ with enzymic mediated hydrolysis. The main goal of this approach was to improve 
drug delivery by overcoming the water solubility problems of the parent drugs, and to enhance their corneal penetration. The PGF2 $\alpha$-TML codrug as well as the PGF2 $\alpha$-ATL codrug successfully underwent hydrolysis in vitro to generate PGF2 $\alpha$ and TML or ATL, respectively in phosphate buffer pH 7.4 and in human serum [68].

\section{Enzyme Inhibitors to Modulate Prodrugs transport and metabolism}

Utility of enzyme inhibitors to modulate transport and metabolism of prodrugs appears to be promising strategy for enhancing drug transport across cornea. Katragadda et al. defined the principle enzyme classes responsible for the hydrolysis of the $L$-valine ester of acyclovir, valacyclovir (VACV) and the L-glycine-valine ester of acyclovir, gly-val-acyclovir (GVACV) as carboxylesterases and aminopeptidases, respectively. Hydrolysis studies of VACV and GVACV in corneal homogenate were conducted in presence of various enzyme inhibitors. Complete inhibition of VACV hydrolysis was observed in the presence of 4-(2-aminoethyl)benzenesulfonyl fluoride and $p$ chloromercuribenzoic acid. Similar trend was also observed with GVACV in the presence of bestatin [69].

Thus, the prodrug approach offers a wide range of options in drug design and delivery. However, to successfully implement these strategies it is important to have an understanding of the distribution of the various metabolic enzymes in ocular tissues. The acyclovir prodrugs, valacyclovir and valvalacyclovir were observed to be substrates of the peptide transporter expressed on the corneal tissue, and demonstrated greater permeability when compared to the parent drug [70].

In an attempt to enhance corneal drug absorption through prodrug modification targeted to the amino acid transporters, Balakrishnan et al. carried out uptake studies using $\left[{ }^{3} \mathrm{H}\right] \mathrm{L}$-tyrosine at $37{ }^{\circ} \mathrm{C}$ and showed a major saturable and minor non-saturable component. Competitive inhibition studies were performed in the presence of various amino acids and model tyrosine conjugates (i.e. $p$-nitro and $p$-chloro benzyl ether conjugates of $L$-tyrosine). Uptake was inhibited by neutral aromatic and large neutral aliphatic amino acids. L-Tyrosine uptake was inhibited by its ether conjugates in a concentration- dependent manner suggesting that these compounds may be sharing the same transport mechanism [71].

Transporter-targeted prodrug derivatization was investigated also utilizing quinidine, a model Pglycoprotein (P-gp) substrate. Results clearly indicated that the $L$-valine and $L$-valine-valine prodrug esters of quinidine can modulate P-gp-mediated efflux and enhance permeability [72]. These prodrugs have a reduced or diminished affinity toward P-gp and were further recognized by the peptide transporter-mediated process. Enhanced permeability of the prodrugs indicates that prodrugs can be a viable strategy for overcoming P-gp-mediated efflux. Furthermore, Majumdar et al. studied a series of dipeptide monoester ganciclovir (GCV) prodrugs; Val-Val-GCV, Tyr-Val-GCV, and Gly-Val-GCV and reported that the transcorneal permeability of Val-GCV and Val-Val-GCV was seven- to eight fold greater than that of GCV, in the presence of a proton gradient, and was significantly decreased in the presence of Gly-Pro. Val-Val-GCV (1\% w/v) provided significantly better therapeutic activity than trifluorothymidine $(1 \% \mathrm{w} / \mathrm{v})$ against HSV-1 epithelial keratitis, and equivalent therapeutic activity against stromal keratitis in the rabbit eye model. It was found that Val-Val-GCV afforded excellent 
corneal permeability and chemical stability, high aqueous solubility, and substantial in vivo antiviral activity against HSV-1 [73].

\section{Eye Irritation and Corneal Damage Assessment}

Different methodologies were used to assess and evaluate the eye irritation due to topically applied prodrugs. Ashton et al. synthesized a group of polyethylene glycol flurbiprofenate prodrugs and reported them to cause no corneal damage when tested in albino rabbit eyes and measured by differential scanning calorimetry [74]. The eye irritation of the water soluble Cyclosporine A prodrug (UNIL-088), a novel Cyclosporine A ester with $N$-[[1-(acetyloxy)ethoxy]carbonyl]-O-phosphono- $L$ seryl- $N$-methylglycine was assessed by the Draize test and by Confocal Laser Ophthalmoscopy (CLSO). The prodrug showed an excellent tolerance for the prodrug solution compared to reference cyclosporine oil solution which induced lachrymation and irritation [75].

\section{Conclusions}

Separation of therapeutic effect from toxicity is a valuable goal in the drug development process. Careful consideration and understanding of ocular tissue metabolic processes within the eye has important implications for controlling the detoxification of therapeutic agents, and for providing the potential for site-specific bio-activation of certain prodrugs and codrugs, thus enabling significant improvements in efficacy and the minimization of local and systemic side-effects. The prodrug and codrug approaches outlined in this review article may provide relevant information to researchers involved in designing appropriate and effective delivery systems for the treatment of ocular disorders.

\section{References and Notes}

1. Maurice, D.M.; Mishima, S. Ocular pharmacokinetics. Handbook of Experimental Pharmacology; Springer Verlag: Berlin-Heidelberg, 1984; 69 (Pharmacol. Eye), pp. 19-116.

2. Visor, G.C. Drug design strategies for ocular therapeutics. Adv. Drug Deliv. Rev. 1994, 14, 269279.

3. Geroski, D.H.; Edelhauser, H.F. Transscleral drug delivery for posterior segment disease. Adv. Drug Deliv. Rev. 2001, 52, 37-48.

4. Duvvuri, S.; Majumdar, S.; Mitra, A.K. Drug delivery to the retina: challenges and opportunities. Expert Opin. Biol. Ther. 2003, 3, 45-56.

5. Duvvuri, S.; Majumdar, S.; Mitra, A.K. Role of metabolism in ocular drug delivery. Curr. Drug Metab. 2004, 5, 507-515.

6. Bodor, N.; Buchwald, P. Ophthalmic drug design based on the metabolic activity of the eye: soft drugs and chemical delivery systems. AAPS J. 2005, 7, 4.

7. Guyto, A.C. Textbook of Medical Physiology, $8^{\text {th }}$ Ed.; W.B. Saunders Co.: Philadelphia, PA, 1991.

8. Leinweber, F.J. Drug Disposition in the mammalian eye and brain: a comparison of mechanisms. Drug Metab. Rev. 1991, 23, 133-246.

9. Moorthy, R..; Valluri, S. Ocular toxicity associated with systemic drug therapy. Curr. Opin Ophthalmol. 1999, 10, 438-46. 
10. Jaanus, S.D. Ocular side effects of selected systemic drugs. Optom Clin. 1992, 2, 73-96.

11. Bourlais, C.L.; Acar, L.; Zia, H.; Sado, P.A.; Needham, T.; Leverge, R. Ophthalmic drug delivery systems recent advances. Prog. Retin. Eye Res. 1998, 17, 33-58.

12. Kishida, K.; Matsumoto, K.; Manabe, R.; Sugiyama, T. Cytochrome P450 and related components of the microsomal electron transport system in the bovine ciliary body. Curr. Eye Res. 1986, 5, 529-533.

13. Schwartzman, M.L.; Abraham N.G.; Masferrer, J.; Dunn, W.; Mcgiff, J.C. Cytochrome P450 dependent metabolism of arachidonic acid in bovine corneal epithelium. Biochem. Biophys. Res. Comm. 1985, 132, 343-351.

14. Schwartzman, M.L.; Masferrer, J.: Dunn, M.W.; Mcgiff, J.C.; Abraham, N.G. Cytochrome P450, drug metabolizing enzymes and arachidonic acid metabolism in bovine ocular tissues. Curr. Eye Res. 1987, 6, 623-630.

15. Asakura, T.; Shishi, H.. Cytochrome P450-mediated prostaglandin omega/omega-1 hydroxylase activities in porcine ciliary body epithelial cells. Exp. Eye Res. 1992, 55, 377-384.

16. Shichi, H.; Atlas, S.A.; Nebert, D.W. Genetically regulated arylhydrocarbon hydroxylase induction in the eye: possible significance of the drug-metabolising enzyme system for the retinal pigmented epithelium-choriod. Exp. Eye Res. 1975, 21, 557-567.

17. Shichi, H.; Tsunematsu, Y.; Nebert, D.W. Aryl hydrocarbon hydroxylase induction in retinal pigmented epithelium: possible associated of genetic differences in a drug-metabolizing enzyme system with retinal degeneration. Exp. Eye Res. 1976, 23, 165-176.

18. Schwartzman, M.L.; Pageno, P.J.; Mcgriff, J.C.; Abraham, N.G. Immunochemical studies on the contribution of NADPH cytochrome P450 reductase to the cytochrome P450 dependent metabolism of arachidonic acid. Arch. Biochem. Biophys. 1987, 252, 635-645.

19. Shichi, H.; Nebert, D.W. Generic differences in drug metabolism associated with ocular toxicity. Envir. Health Perspec. 1982, 44, 107-117.

20. Sakamoto, S; Shichi, H. Induction of alkoxyresorufin O-dealkylases and UDP-glucuronosyl transferase by Phenobarbital and 3-methylcholanthrene in primary cultures of porcine ciliary epithelial cells. Biochem. Pharmacol. 1991, 41, 611-616.

21. Shanthaveerappa, T.R.; Bourne, G.H. Monoamine oxidase distribution in the rabbit eye. J. Histochem. Cytochem. 1964, 12, 281-287.

22. Sparks, D.L.; Thomas, T.N.; Buckholtz, N.S. Monoamine oxidase activity in bovine retina: subcellular distribution and drug sensitivities. Neurosci. Lett. 1981, 21, 201-6.

23. Crabbe, M.J.C. Ocular diamine oxidase activity. Exp. Eye Res. 1985, 41, 777-778.

24. Kremzner, L.T.; Roy, D.; Spector, A. Polyamines in normal and cataractous human lenses. Evidence for post-translational modifications. Exp. Eye Res. 1983, 37, 649-659.

25. Lee, V.H.L. Esterase activities in adult rabbit eyes. J. Pharm. Sci. 1983, 72, 239-244.

26. Sanchez-Chavez, G.; Vidal, C. J.; Salceda, R. Acetyl- and butyrylcholinesterase activities in the rat retina and retinal pigment epithelium. J. Neurosci. Res. 1995, 41, 655-62.

27. Dutton, G.J. Glucuronidation of Drugs and Other Compounds; CRC Press: Boca Raton, FL, 1966.

28. Coupland, S.E.; Penfold, P.L.; Billson, F.A. Hydrolases of anterior segment tissues in the normal human, pig and rat eye: a comparative study. Arch. Clin. Exp. Ophthalmol. 1994, 232, 182-92. 
29. Coupland, S.E.; Hoffman, H.H.; Penfoldand, P.L.; Billson, F.A. Increased hydroxylase activities in the human trabecular meshwork of glaucomatous eyes. Ger. J. Ophthalmol. 1993, 2, 107-112.

30. Physlactos, A.C. The lysosomal enzymes of the iris-ciliary body are retained in their organelles and exhibit increased activities during acute uveal inflamation. Acta Ophthamol. 1991, 69, 33-38.

31. Rao, P.V.; Zigler, Jr., J.S. Zeta-crystallin from guinea pig lens is capable of functioning catalytically as an oxidoreductase. Arch. Biochem. Biophys. 1991, 284, 181-185.

32. Gondhowiardojo, T.D.; van Haeringen, N.J.; Hoekzema, R.; Pels, L.; Kijlstra, A. Detection of aldehyde dehydrogenase activity in human corneal extracts. Curr. Eye Res. 1991, 10, 1001-1007.

33. Godbout, R. High levels of aldehyde dehydrogenase transcripts in the undifferentiated chick retina. Exp. Eye Res. 1992, 54, 297-305.

34. Holmes, R.S.; Vandeberg, J.L. Ocular NAD-dependent alcohol dehydrogenase and aldyhyde dehydrogenase in the baboon. Exp. Eye Res. 1986, 43, 383-398.

35. Watkins, J.; Wirthwein, D.P.; Sanders, R.A. Comparative study of phase II biotransformation in rabbit ocular tissues. Drug Metab. Dispos. 1991, 19, 708-13.

36. Saneto, R.P.; Awasthi, Y.C.; Srivastava, S.K. Mercapturic acid pathway enzymes in bovine ocular lens, cornea, retina and retinal pigmented epithelium. Exp. Eye Res. 1982, 34, 107-111.

37. Saneto, R.P.; Awasthi, Y.C.; Srivastava, S.K. Glutathione S-transferases of the bovine retina: evidence that glutathione peroxidase activity is the result of glutathione S-transferase. Biochem. J. 1982, 205, 213-217.

38. Ahmed, H.; Singh, S.V.; Medh, R.D.; Ansari, G.A.S.; Kurosoku, A.; Awasthi, Y.C. Differential expression of Omega, Beta, and pi classes isozymes of glutathione S-transferase in bovine lens, cornea and retina. Arch. Biochem. Biophys. 1988, 266, 416-426.

39. Miller,L.; Stier, M.; Lovenburg, W. Evidence for the presence of N-acetyl transferase in the rat retina. Comp. Biochem. Physiol. 1980, 66, 213-216.

40. Sastry, B.V.; Vidaver, P.S.; Janson, V.E.; Franks, J.J. S-adenosyl-L-methionine-mediated enzymatic methylations in the rat retinal membranes. J. Ocular Pharmacol. 1994, 10, 253-263.

41. Maurice, D.M.; Mishima, S., Pharmacology of the eye. In: Handbook of experimental pharmacology; Sears, M.L. (Ed.); Springer-Verlag: Berlin, Heidelberg, 1984; pp. 16-119.

42. Mosher, G.L.; Mikkelson, T.J. Permeability of the $N$-alkyl $p$-aminobenzoate esters across the isolated corneal membrane of the rabbit. Int. J. Pharm. 1979, 2, 239-243.

43. Bundgaard, H.; Falch, E.; Larsen, C.; Mosher, G. L.; Mikkelson, T. J. Pilocarpic acid esters as novel sequentially labile pilocarpine prodrugs for improved ocular delivery. J. Med. Chem. 1985, 28, 79-81.

44. Bundgaard, H.; Falch, E.; Larsen, C.; Mosher, G. L.; Mikkelson, T. J. Pilocarpine prodrugs. II. Synthesis, stability, bioconversion, and physicochemical properties of sequentially labile pilocarpine acid diesters. J. Pharm. Sci. 1986, 75, 775-83.

45. Druzgala, P.l; Winwood, D.; Drewniak-Deyrup, M.; Smith, S.; Bodor, N.; Kaminski, J.J. New water-soluble pilocarpine derivatives with enhanced and sustained muscarinic activity. Pharm. Res. 1992, 9, 372-7.

46. Druzgala, P.; Bodor, N. Water soluble pilocarpine prodrugs with sustained intraocular activity in normotensive rabbits and in glaucomatous beagles. J. Control. Release 1994, 28, 282-3. 
47. Thombre, A.G.; Himmelstein, K.J. Quantitative evaluation of topically applied pilocarpine in the precorneal area. J. Pharm. Sci. 1984, 73, 219-22.

48. Meseguer, G.; Gurny, R.; Buri, P.; Rozier, A.; Plazonnet, B. Gamma scintigraphic study of precorneal drainage and assessment of miotic response in rabbits of various ophthalmic formulations containing pilocarpine. Int. J. Pharm. 1993, 95, 229-34.

49. Patton, T.F. Pharmacokinetic evidence for improved ophthalmic drug delivery by reduction of instilled volume. J. Pharm. Sci. 1977, 66, 1058-9.

50. Bundgaard, H.; Falch, E.; Larsen, C.; Mikkelson, T.J. Pilocarpine prodrugs I. Synthesis, physicochemical properties and kinetics of lactonization of pilocarpic acid esters. J. Pharm. Sci. 1986, 75, 36-43.

51. Suhonen, P.; Jarvinen, T.; Peura, P.; Urtti, A. Permeability of pilocarpic acid diesters across albino rabbit cornea in vitro. Int. J. Pharm. 1991, 74, 221-8.

52. Saarinen-Savolainen, P.; Jaervinen, T.; Suhonen, P.; Urtti, A. Amphiphilic properties of pilocarpine prodrugs. Int. J. Pharm. 1996, 133, 171-178.

53. Suhonen, P.; Jaervinen, T.; Lehmussaari, K.; Reunamaeki, T.; Urtti, A. Ocular absorption and irritation of pilocarpine prodrug is modified with buffer, polymer, and cyclodextrin in the eyedrop. Pharm. Res. 1995, 12, 529-33.

54. Jarho, P.; Jaervinen, K.; Urtti, A.; Stella, V.J.; Jaervlinen, T. Modified $\beta$-cyclodextrin (SBE7- $\beta$ CyD) with viscous vehicle improves the ocular delivery and tolerability of pilocarpine prodrug in rabbits. J. Pharm. Pharmacol. 1996, 48, 263-269.

55. Sznitowska, M.; Zurowska-Pryczkowska, K.; Janicki, S.; Jarvinen, T. Miotic effect and irritation potential of pilocarpine prodrug incorporated into a submicron emulsion vehicle. Int. J. Pharm. 1999, 184, 115-120.

56. Chrai, S.S.; Patton, T.F.; Mehta, A.; Robinson, J.R. Lacrimal and instilled fluid dynamics in rabbit eyes. J. Pharm. Sci. 1973, 62, 1112-1121.

57. Himmelstein, K.J.; Guvenir, I.; Patton, T.F. Preliminary pharmacokinetic model of pilocarpine uptake and distribution in the eye. J. Pharm. Sci. 1978, 67, 603-606.

58. Van Buskirk, E.M. Adverse reactions from timolol administration. Ophthalmology 1980, 87, 447450.

59. Nelson, W.L.; Fraunfelder, F.T.; Sills, J.M.; Arrowsmith, J.B.; Kuritsky, J.N. Adverse respiratory and cardiovascular events attributed to timolol ophthalmic solution, 1978-1985. Am. J. Ophthalmol. 1986, 102, 606-611.

60. Sasaki, H.; Igarashi, Y.; Nishida, K.; Nakamura, J. Ocular delivery of the $\beta$-blocker, tilisolol, through the prodrug approach. Int. J. Pharm. 1993, 93, 49-60.

61. Hellberg, M.R.; Ke, T-L.; Haggard, K.; Klimko, P.G.; Dean, T.R.; Graff, G. The Hydrolysis of the Prostaglandin Analog Prodrug Bimatoprost to 17-Phenyltrinor PGF2 $\alpha$ by Human and Rabbit Ocular Tissue. J. Ocular Pharm. Therapeut. 2003, 19, 97-103.

62. Hussain, A.; Truelove, J. E. Prodrug approaches to enhancement of physicochemical properties of drugs. IV. Novel epinephrine prodrug. J. Pharm. Sci. 1976, 65, 1510-12.

63. Howard-Sparks, M.; Al-Ghananeem, A.M.; Pearson, A.P.; Crooks, P.A. Evaluation of O3 $\alpha-$, O21Di-(N1-methyloxycarbonyl-2,4-dioxo-5-fluoropyrimidinyl)17 $\alpha$-hydroxy-5 $\beta$-pregnan-20-one as a novel potential antiangiogenic codrug. J. Enzyme Inhib. Med. Chem. 2005, 20, 417-428. 
64. Hamad, M.O.; Kiptoo, P.K.; Stinchcomb, A.L.; Crooks, P.A. Synthesis and hydrolytic behavior of two novel tripartate codrugs of naltrexone and 6 6 -naltrexol with hydroxybupropion as potential alcohol abuse and smoking cessation agents. Bioorg. Med. Chem. 2006, 14, 7051-7061.

65. Howard-Sparks, M.; Al-Ghananeem, A.; Pearson, A.; Crooks, P. A Novel Chemical Delivery System Comprising an Ocular Sustained Release Formulation of a $3 \alpha, 17 \alpha, 21$-trihydroxy-5 $\beta$ pregnan-20-one-BIS-5-Flourouracil Codrug. Drug Dev. Ind. Pharm. In Press.

66. Cardillo, J.A.; Farah, M.E.; Mitre, J.; Morales, P.H.; Costa, R.A.; Melo, L.A.S.; Kuppermann, B.; Jorge, R.; Ashton, P. An intravitreal biodegradable sustained release naproxen and 5-fluorouracil system for the treatment of experimental post-traumatic proliferative vitreoretinopathy. $\mathrm{Br} . \mathrm{J}$. Ophthalmol. 2004, 88, 1201-5.

67. Cynkowska, G.; Cynkowski, T.; Al-Ghananeem, A.M.; Al-Ghananeem, A.A.; Guo, H.; Ashton, P.; Crooks, P.A. Novel antiglaucoma prodrugs and codrugs of ethacrynic acid. Bioorg. Med. Chem Lett. 2005, 15, 3524-7.

68. Cynkowska, G.; Cynkowski, T.; Guo, H.; Ashton, P.; Crooks, P. A. Synthesis of novel codrugs of prostaglandin F2 $\alpha$ with $\beta$-adrenergic receptor blockers for the treatment of ocular diseases. Book of Abstracts, 211th ACS National Meeting; New Orleans, LA, March 24-28, 1996.

69. Katragadda, S.; Talluri, R.S.; Mitra, A.K. Simultaneous modulation of transport and metabolism of acyclovir prodrugs across rabbit cornea: An approach involving enzyme inhibitors. Int. J. Pharm. 2006, 320, 104-113.

70. Anand, B.S.; Mitra, A.K. Mechanism of Corneal Permeation of L-Valyl Ester of Acyclovir: Targeting the Oligopeptide Transporter on the Rabbit Cornea. Pharm. Res. 2002, 19, 1194-1202.

71. Balakrishnan, A.; Jain-Vakkalagadda, B.; Yang, C.; Pal, D.; Mitra, A.K. Carrier mediated uptake of L-tyrosine and its competitive inhibition by model tyrosine linked compounds in a rabbit corneal cell line (SIRC)-strategy for the design of transporter/receptor targeted prodrugs. Int. J. Pharm. 2002, 247, 115-125.

72. Katragadda, S.; Talluri, R.S.; Mitra, A.K. Modulation of P-glycoprotein-mediated efflux by prodrug derivatization: an approach involving peptide transporter-mediated influx across rabbit cornea. J. Ocul. Pharmacol. Ther. 2006, 22, 110-120.

73. Majumdar, S.; Nashed, Y. E.; Patel, K.; Jain, R.; Itahashi, M.; Neumann, D. M.; Hill, J. M.; Mitra, A. K. Dipeptide Monoester Ganciclovir Prodrugs for Treating HSV-1-Induced Corneal Epithelial and Stromal Keratitis: In Vitro and In Vivo Evaluations. J. Ocul. Pharmacol. Ther. 2005, 21, 463474.

74. Ashton, P.; Smith, T.J.; Glavinos, P.G.; Conklin, Jr., J.D.; Crooks, P.A.; Riggs, R.M.; Cynkowski, T.; Cynkowska, G. Permeable, non-irritating prodrugs of nonsteroidal and steroidal agents. U.S. Pat. 5,681,964, 1997.

75. Lallemand, F.; Furrer, P.; Felt-Baeyens, O.; Gex-Fabry, M.; Dumont, J.-M.; Besseghir, K.; Gurny, R. A novel water-soluble cyclosporine A prodrug: ocular tolerance and in vivo kinetics. Int. J. Pharm. 2005, 295, 7-14.

(C) 2007 by MDPI (http://www.mdpi.org). Reproduction is permitted for noncommercial purposes. 